\title{
Effects of lifestyle intervention on dietary intake, physical activity level, and gestational weight gain in pregnant women with different pre-pregnancy Body Mass Index in a randomized control trial
}

\author{
Amy Leung Hui ${ }^{1 *}$, Lisa Back', Sora Ludwig ${ }^{1}$, Phillip Gardiner², Gustaaf Sevenhuysen ${ }^{3}$, Heather J Dean ${ }^{4}$, \\ Elisabeth Sellers ${ }^{4}$, Jonathan McGavock ${ }^{4}$, Margaret Morris ${ }^{5}$, Depeng Jiang ${ }^{1}$ and Garry X Shen ${ }^{1}$
}

\begin{abstract}
Background: The objectives of this study were to assess the efficacy of lifestyle intervention on gestational weight gain in pregnant women with normal and above normal body mass index (BMI) in a randomized controlled trial.
\end{abstract}

Methods: A total of 116 pregnant women ( $<20$ weeks of pregnancy) without diabetes were enrolled and 113 pregnant women completed the program. Participants were randomized into intervention and control groups. Women in the intervention group received weekly trainer-led group exercise sessions, instructed home exercise for 3-5-times/week during 20-36 weeks of gestation, and dietary counseling twice during pregnancy. Participants in the control group did not receive the intervention. All participants completed a physical activity questionnaire and a 3-day food record at enrolment and 2 months after enrolment.

Results: The participants in the intervention group with normal pre-pregnancy BMI $(\leq 24.9 \mathrm{~kg} / \mathrm{M} 2, \mathrm{n}=30)$ had lower gestational weight gain (GWG), offspring birth weight and excessive gestational weight gain (EGWG) on pregnancy weight gain compared to the control group $(n=27, p<0.05)$. Those weight related-changes were not detected between the intervention $(n=27)$ and control group $(n=29)$ in the above normal pre-pregnancy BMl participants. Intervention reduced total calorie, total fat, saturated fat and cholesterol intake were detected in women with normal or above normal pre-pregnancy BMI compared to the control group ( $<<0.05$ or 0.01 ). Increased physical activity and reduced carbohydrate intake were detected in women with normal $(p<0.05)$, but not above normal, pre-pregnancy $\mathrm{BMl}$ at 2 months after the onset of the intervention compared to the control group.

Conclusion: The results of the present study demonstrated that the lifestyle intervention program decreased EGWG, GWG, offspring birth weight in pregnant women with normal, but not above normal, pre-pregnancy BMI, which was associated with increased physical activity and decreased carbohydrate intake.

Trial registration: NCT00486629

Keywords: Pregnancy, Gestational diabetes, Nutrition, Pregnancy weight gain

\footnotetext{
* Correspondence: Amy.Hui@med.umanitoba.ca

'Departments of Internal Medicine, University of Manitoba, Room 801D, 715

McDermot Ave., Winnipeg, MB R3E 3P4, Canada

Full list of author information is available at the end of the article
} 


\section{Background}

Obesity has been recognized as a health issue, which increases risk for several common chronic diseases [1-3]. The guidelines of the Canadian Medical Association Institute for the management and prevention of obesity have recommended the measurement of both body mass index (BMI) and waist circumference to assess the level and distribution of adiposity in adults [4]. BMI was calculated as weight in kilograms divided by height in meters squared. Health Canada defines underweight as a BMI less than $18.5 \mathrm{~kg} / \mathrm{m}$ [2], normal weight as a BMI of 18.5-24.9, overweight as a BMI of 25.0-29.9, class I obesity as a BMI of 30.0 - 34.9, class II obesity as a BMI of $35.0-39.9$, and class III obesity as a BMI $\geq 40.0$ [1]. In Canada, it was estimated that approximately 8.6 million of adults with age $>18$ years were overweight and 5.5 million were obese in 2005 [5]. The rising prevalence of obesity has increased the percentage of obesity in women at childbearing age in Canada. The 2006-2007 Canadian Maternal Experience Survey estimated that approximately $23 \%$ and $18 \%$ of the women began their pregnancy as overweight or obese [6]. Pre-pregnancy BMI $\geq 25$ or above normal BMI increases the risk of poor outcomes of pregnancy including gestational diabetes, preeclampsia, hypertension and cesarean section [7].

In 2009, the Institute of Medicine (IOM) revised the 1990 guidelines of recommended weight gain during pregnancy in response to the worldwide epidemic of obesity and the demand to reduce obesity [7]. The guidelines have been endorsed by the American College of Obstetricians and Gynecologists [8] and the Health Canada [9]. Behavioral interventions such as weight awareness and dietary pattern improvement may mitigate the risks of pregnancy complications. Several studies examined the impact of lifestyle interventions (dietary intervention with or without added physical activity) on excessive gestational weight gain (EGWG) using the IOM 2009 guidelines. The results of those studies, either randomized controlled trials (RCT) or clinical studies, were not homogenous [10-19]. We hypothesize that normal weight and above normal weight pregnant women may have different responses to a lifestyle intervention in terms of gestational weight gain (GWG).

In order to test this hypothesis, we examined the impact of a lifestyle intervention program on pregnant women in normal and above normal pre-pregnancy BMI categories. EGWG, physical activity levels, dietary intake were compared between the control and intervention groups in each BMI category via a RCT.

\section{Methods}

\section{Subjects}

This study recruited 116 pregnant women who lived in Winnipeg, Manitoba between May 2009 and December
2011. This sample size was based on two previous studies that using Pre-pregnancy BMI subgroups and detected significant gestational weight gain difference between two BMI groups $[13,16]$. Inclusion criteria were: less than 20 weeks of pregnancy, no existing diabetes during pregnancy and signed consent form. These participants were recruited from prenatal classes or community clinics through posters or local newspaper advertisements in Winnipeg. The study protocol and consent form were approved by the Research Ethics Board of the University of Manitoba. Three applicants were excluded from the study because of the existence of medical or obstetric contraindication for exercise during pregnancy. One hundred and thirteen eligible participants were randomized into control or intervention group (Figure 1). Randomization was performed using a computer-generated randomization allocation table by a staff member without involvement in the study design. After randomization, participants received a sealed envelope labelled with the assigned randomization number, which contained instructions for participants. The nature of the study meant that participants and study staff were not blinded to the types of interventions. None of the participants discontinued during the participation. No complain to the program was reported by the participants.

\section{Intervention program \\ Instructed exercise}

Participants in the intervention group received communitybased weekly exercise program which was developed in our previous studies $[19,20]$. The exercise program included mild-to-moderate aerobic exercise, stretching, and strength exercise, and was delivered in weekly group exercise class or a DVD format to assist home exercise. Participants were encouraged to exercise for 3-5 times a week, 30-45 minutes/ time, including attending group exercise class or following the exercise DVD instruction at home. The exercise intervention period was from 20-26 gestational weeks to 36 gestational weeks. Participants kept a logbook on their exercise activities as a motivator for exercise. Attendance less than 3 times at the group exercise, showed no interest to exercise at home or no record of exercise in logbook were considered as withdraw from the study.

\section{Dietary intervention}

Participants in the intervention group received one-onone private dietary consultation at baseline and at two months after. The dietary consultation was performed using a Food Choice Map (FCM) software. The FCM has been proved to be a valid tool for assessing dietary intake [21]. During the consultation, participants recalled their food intakes in a typical week. Participants and dietitians jointly placed food stickers on a magnetic board. The 


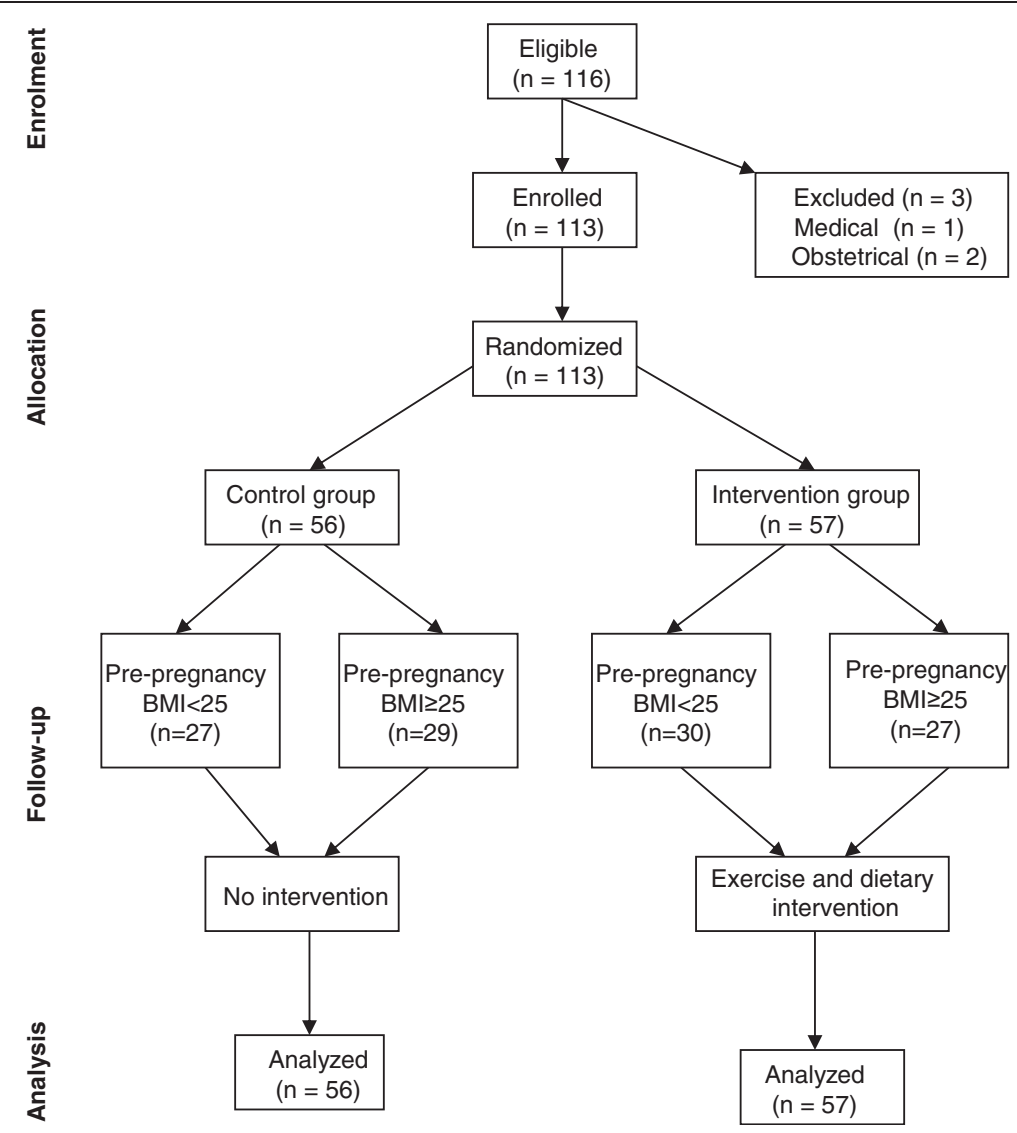

Figure 1 Consort flow chart of the study.

nutritional information on the food sticker, including food items, portion sizes, the frequency of each food, was scanned into the computer at the end of each interview [22]. Daily calorie intake and macronutrients were analyzed instantly. Nutritional recommendations were based on the dietary intake analysis and Health Canada guidelines for food intake in pregnancy $[23,24]$ with considerations on personal food preference, food beliefs, and food budgeting. Weight gain goal was discussed and emphasized through consultation. FCM is an effective way to identify factors that are relevant to a particular health behavior in a population under investigation. Such a tool allows the participants to comment on all the foods that she consumed in real life, without missing or neglecting certain foods cognitively. Results from this kind of data collection could capture the whole picture of food choice decision makings in the participants. Food Choice Map (FCM) interview tool provided such an opportunity to obtain a complete weekly intake and reasons behind food choices. This approach is unique in the literature. Because of this approach, the nutrition intervention was not simply making dietary assessment and deliver education, but to create a personalized, achievable dietary plan with consideration of participants reasons for food choice decision making. A copy of the FCM with agreed changed written on the copy was given to the participant. This copy was served as the diet plan to promote dietary behavior changes. A follow-up dietary consultation was performed at 2 months later to reinforce the recommendations.

\section{Control group}

Participants in the control group did not receive the exercise and dietary interventions. Participants in the control group received standard prenatal care recommended as by the Society of Obstetricians and Gynecologists of Canada. They were also provided with a package of current information on physical activity and healthy eating during pregnancy from the Health Canada [25].

\section{Data collection}

Data on delivery route, maternal weight at delivery room, birth weight and birth weight-related obstetric procedures (induction, forceps or caesarean section) were collected from hospital medical charts by student assistants without knowledge in study design. Diagnosis of 
gestational diabetes was done by the participant's attending health care team according to the 2008 guidelines of the Canadian Diabetes Association [26]. Large-forgestational-age was determined based on birth weight and gestational age as previously described [27]. Prepregnancy weights of participants, height and BMI were obtained from the Manitoba Prenatal Care Record. If the pre-pregnancy weight was missing on the record, the weight at the first contact of study participation (less than 10 weeks gestation) was used as pre-pregnancy weight. GWG was defined as maternal weight at delivery subtracts pre-pregnancy weight. EGWG was calculated by subtracting the upper limit of normal weight gain for corresponding pre-pregnancy BMI according to the 2009 guidelines of IOM [7] from the actual weight gain (difference between pre-pregnancy weight and bodyweight at delivery room).

Physical activity levels at the enrolment and 2 months thereafter were assessed subjectively in all participants using a PARMed-X form for Pregnancy designed by the Canadian Society of Exercise Physiology, which was validated previously using peak oxygen consumption [28]. Unfit (physical activity index $=0$ ) during pregnancy was defined as recreational activity $<1-2$ times/week plus $<20$ minutes/time. Active (physical activity index $=1$ ) was defined as recreational activity 1-2 times/week, $>20$ minutes/time or $>2$ times/week but $<20$ minutes/time. Fit (physical activity index $=2$ ) was defined as recreational activity $>2$ times/week plus $>20$ minutes/time [19].

Food intakes of all participants were assessed using 3-day food records at enrolment and 2 months after the enrolment [29]. The results of self-reported food intake were analyzed using NutriBase 6.0 software containing Canadian Food Database (Cyber- Soft, Inc., Phoenix, AZ, USA).

\section{Statistical methods}

The statistical analyses were performed by a third party. Quantitative data were expressed in mean \pm SD. The comparisons for continuous data between 2 groups were conducted using the Student t-test. Categorical data were analyzed using non-parametric Fisher's exact test. The significant difference was pre-set at $\mathrm{p}<0.05$.

\section{Results}

One hundred and thirteen participants (56 in the control group and 57 in the intervention group) completed their program and delivered babies before December 31, 2011. All participants in the intervention group met with the dietitian at baseline and at 2 months after. These women attended the group exercise and exercise regularly at home according to the protocol. No withdraw from both intervention and control groups. In the intervention group, 30 women had their pre-pregnancy BMI $\leq 24.9$, and 27 women had their pre-pregnancy BMI $\geq 25$. In the control group, 27 women had their pre-pregnancy BMI $\leq 24.9$ and 29 women had pre-pregnancy BMI $\geq 25$. No significant difference was detected in pre-pregnancy BMI, the proportions of First Nations women, or annual family income between the control and intervention groups (Table 1).

In the normal pre-pregnancy BMI subgroups, the amount of GWG was approximately $20 \%$ lower in the intervention group compared to that in the control group (16.23 \pm $4.38 \mathrm{~kg}$ vs. $12.9 \pm 3.72 \mathrm{~kg}, \mathrm{p}<0.05)$. The rate of EGWG was significantly lower in the intervention group compared to that in the control group $(10 \%$ versus $37 \%, \mathrm{p}<0.05)$. Birth weights of offspring of participants in the intervention group were significantly lower than that in the control group (3 $633 \pm 555$ g vs. $3356 \pm 474 \mathrm{~g}, \mathrm{p}<0.05)$.

Table 1 Demographic and outcome data

\begin{tabular}{|c|c|c|c|c|c|c|}
\hline \multirow[b]{2}{*}{ Variables } & \multicolumn{3}{|c|}{ Pre-pregnancy BMI $\leq 24.9$} & \multicolumn{3}{|c|}{ Pre-pregnancy $\mathrm{BMI} \geq 25$} \\
\hline & Control $n=27$ & Intervention $\mathrm{n}=\mathbf{3 0}$ & P-value & Control $n=29$ & Intervention $\mathbf{n}=\mathbf{2 7}$ & P-value \\
\hline Age (years) & $29 \pm 6$ & $31 \pm 3$ & 0.06 & $32 \pm 5$ & $31 \pm 4$ & 0.41 \\
\hline Length of intervention (weeks) & 0 & $27.83 \pm 5.67$ & & 0 & $26.74 \pm 6.17^{*}$ & \\
\hline Family annual income (\$) & $54,404 \pm 33,689$ & $53,564 \pm 24,128$ & 0.91 & $50,992 \pm 23,199$ & $56,772 \pm 26,355$ & 0.39 \\
\hline First Nations (number \%) & $1 / 27$ & $2 / 30$ & 0.62 & $4 / 29$ & $3 / 27$ & 0.92 \\
\hline Pre-pregnancy BMI & $22.6 \pm 1.9$ & $21.6 \pm 2.2$ & 0.06 & $29.7 \pm 1.3$ & $29.5 \pm 5.1$ & 0.92 \\
\hline Gestational weeks (week) & $39.6 \pm 0.9$ & $39.7 \pm 1.1$ & 0.78 & $39.8 \pm 1.1$ & $39.7 \pm 1.3$ & 0.92 \\
\hline Gestational weight gain (kg) & $16.23 \pm 4.38$ & $12.9 \pm 3.72$ & 0.03 & $14.39 \pm 7.05$ & $15.21 \pm 7.5$ & 0.26 \\
\hline EGWG (2009 IOM guidelines number \%) & 10/27 (37\%) & $3 / 30(10 \%)$ & 0.03 & $20 / 29(69 \%)$ & $18 / 27(67 \%)$ & 0.67 \\
\hline Birth weight (g) & $3,633 \pm 555$ & $3,356 \pm 474$ & 0.047 & $3650 \pm 481$ & $3,665 \pm 506$ & 0.92 \\
\hline Large-for-gestational-age (n \%) & $3 / 27(11 \%)$ & $2 / 30(7 \%)$ & 0.902 & $1 / 29(3 \%)$ & $4 / 27(15 \%)$ & 0.13 \\
\hline Gestational diabetes (n \%) & $0 / 27$ & $0 / 30$ & NS & $3 / 29(10 \%)$ & $1 / 27(4 \%)$ & 0.307 \\
\hline Cesarean section (n \%) & $0 / 27$ & $0 / 30$ & NS & $2 / 29(7 \%)$ & $0 / 27$ & 0.503 \\
\hline
\end{tabular}

Values were expressed in mean \pm SD or case/total $(0 \%)$. P values with underline are statistical significant.

*The $\mathrm{p}$ value between the pre-pregnancy $\mathrm{BMI} \leq 24.9$ intervention group and the Pre-pregnancy $\mathrm{BMI} \geq 25$ intervention group is 0.49 . 
These variables were not significantly different between the intervention and control groups in the above normal pre-pregnancy BMI women. No significant difference was detected in the prevalence of large-for-gestational age baby, gestational diabetes or cesarean section requirement between the intervention and control groups in women with different pre-pregnant women BMI categories (Table 1).

All participants returned food records and physical activity questionnaires at baseline and at 2 months after. At baseline, no significant difference in nutritional intake or physical activity was detected in normal pre-pregnancy BMI women with and without the lifestyle intervention. The lifestyle intervention significantly improved the pattern of nutritional intake in the normal pre-pregnancy BMI participants compared to the control group. Significantly lower daily intakes of total calorie $(2016 \pm 496 \mathrm{kcal}$ vs. $2551 \pm 1044 \mathrm{kcal})$, carbohydrate $(286.3 \pm 80.7 \mathrm{~g}$ vs. $355.2 \pm 147.6 \mathrm{~g})$, total fat $(63.1 \pm 23.2 \mathrm{~g}$ vs. $87.5 \pm 41.6 \mathrm{~g})$, saturated fat $(20.0 \pm 9.5 \mathrm{~g}$ vs. $29.52 \pm 16.7 \mathrm{~g})$, and cholesterol $(225.0 \pm 115.9 \mathrm{mg}$ vs. $340 \pm 224.9 \mathrm{mg})$ were detected in normal pre-pregnancy BMI women who received the lifestyle intervention compared to that in the control group ( $\mathrm{p}<0.03-0.008$, Table 2 ). Among participants with pre-pregnancy $B M I \geq 25$, significantly lower intakes of total calorie (1 $986 \pm 470 \mathrm{kcal}$ vs. $2258 \pm 546 \mathrm{kcal})$, total fat $(65.7 \pm 27.1 \mathrm{~g}$ vs. $83.5 \pm 30.3 \mathrm{~g})$, saturated fat $(20.6 \pm$ $10.3 \mathrm{~g}$ vs. $27.8 \pm 10.6 \mathrm{~g})$ and cholesterol intake (202.0 \pm $104.3 \mathrm{mg}$ vs. $305.7 \pm 215.2 \mathrm{mg}$ ), but not carbohydrate intake, were detected between above normal pre-pregnancy BMI women with and without intervention at 2 months after the onset of the intervention ( $\mathrm{p} \leq 0.05-0.01$, Table 3 ).

At baseline, no significant differences in physical activity level were detected among any group. However, only women with pre-pregnant $\mathrm{BMI} \leq 24.9$ had significantly higher physical activity units at 2 months after the start of the exercise intervention (intervention group: baseline $1.4 \pm 0.81$ versus 2 months after, $1.87 \pm 0.35, \mathrm{p}<0.05)$. No significant difference in physical activity was observed in the above normal pre-pregnant BMI group between baseline and 2 months after (control: baseline $1.70 \pm 0.61$ versus 2 months after $1.56 \pm 0.51$, Figure 2 ).

\section{Discussion}

The results of previous studies on the efficacy of lifestyle interventions on gestational weight gain or EGWG in overweight or obese women were inconsistent. Two studies that were similar to our study design were Polley et al. and Wolff et al. $[13,16]$ Polley et al. reported that education about weight and exercise reduced EGWG in normal weight pregnant women, but not in overweight pregnant women, in a RCT [16]. Wolff et al. described that dietary counseling significantly reduced GWG in obese pregnant women, but did not affect the rate of EGWG between control and intervention group in another RCT [13]. The present RCT demonstrated that pregnant women with normal pre-pregnancy BMI, but not those with above normal pre-pregnancy BMI, had better weight-related pregnancy outcomes including EGWG, GWG and birth weight of offspring following the lifestyle intervention compared to the control group. The results from the present study support the findings that women with above normal pre-pregnancy BMI are relatively resistant to the lifestyle intervention in terms of GWG reported by Polley et al. [13], which is possibly related to the response of the pregnant women to lifestyle education on food intake and physical activity. Normal pre-pregnancy weight appears to be more perceptive to the lifestyle education to improving weight-related pregnancy outcomes during pregnancy. The current study had different results in pregnancy outcomes compared to Wolff's study. However, total calorie, fat, saturated fat, and cholesterol intake reduction were significantly reduced in the above normal pre-pregnancy BMI group; the pre-pregnancy outcome significance may be detected when the sample size increases.

The nutrition intervention component in this study is unique compared to other nutrition interventions reported in the literature, which used newsletters, group education sessions, personal counselling provided calories and nutrients goals [11-18]. The results showed that all participants in the intervention group made dietary changes regardless with pre-pregnancy BMI. The FCM interview approach ensured a complete review and discussion of a weekly eating pattern. The FCM in-depth interview could explore meanings behind the food choices

Table 2 Nutrition data of participants with pre-pregnancy BMI $\leq \mathbf{2 9 . 4}$

\begin{tabular}{|c|c|c|c|c|c|c|}
\hline \multirow[t]{2}{*}{ Daily intake } & \multicolumn{3}{|c|}{ Baseline } & \multicolumn{3}{|c|}{2 months } \\
\hline & Control $(n=27)$ & Intervention $(n=30)$ & $P$ value & Control $(n=27)$ & Intervention $(\mathrm{n}=30)$ & $P$ value \\
\hline Toatal calorie & $2239 \pm 654$ & $1982 \pm 496$ & 0.12 & $2551 \pm 1044$ & $2016 \pm 496$ & 0.01 \\
\hline Carbohydrate (g) & $302.4 \pm 77.7$ & $272.8 \pm 64.1$ & 0.12 & $355.2 \pm 147.6$ & $286.3 \pm 80.7$ & 0.03 \\
\hline Protein (g) & $90.9 \pm 42.8$ & $89.0 \pm 27.4$ & 0.85 & $96.8 \pm 40.7$ & $88.7 \pm 25.1$ & 0.36 \\
\hline Fat (g) & $77.9 \pm 30.4$ & $64.4 \pm 34.5$ & 0.13 & $87.5 \pm 41.6$ & $63.1 \pm 23.2$ & 0.008 \\
\hline Saturated (g) & $26.2 \pm 12.5$ & $21.17 \pm 1.1$ & 0.11 & $29.52 \pm 16.7$ & $20.0 \pm 9.5$ & 0.008 \\
\hline Cholesterol (mg) & $275.4 \pm 182.2$ & $247.6 \pm 114.8$ & 0.49 & $340 \pm 224.9$ & $225.0 \pm 115.9$ & 0.02 \\
\hline
\end{tabular}

Values are expressed in mean \pm SD and analyzed. a: Control versus Intervention at baseline; b: Control versus Intervention at 2 months after enrollment. 
Table 3 Nutrition data of participants with pre-pregnancy BMI $\geq 25$

\begin{tabular}{|c|c|c|c|c|c|c|}
\hline \multirow[t]{2}{*}{ Daily intake } & \multicolumn{3}{|c|}{ Baseline } & \multicolumn{3}{|c|}{2 months } \\
\hline & Control $(n=29)$ & Intervention $(n=27)$ & $P$ value & Control $(n=29)$ & Intervention $(n=27)$ & $P$ value \\
\hline Total calorie & $2089 \pm 517$ & $2204 \pm 693$ & 0.48 & $2258 \pm 546$ & $1986 \pm 470$ & 0.05 \\
\hline Carbohydrate (g) & $280.1 \pm 77.2$ & $303.0 \pm 93.8$ & 0.32 & $294.4 \pm 86.7$ & $278.5 \pm 64.0$ & 0.44 \\
\hline Protein (g) & $91.2 \pm 26.1$ & $86.2 \pm 25.0$ & 0.46 & $92.0 \pm 26.1$ & $83.1 \pm 22.1$ & 0.17 \\
\hline Fat (g) & $68.0 \pm 23.3$ & $77.8 \pm 35.3$ & 0.22 & $83.5 \pm 30.3$ & $65.7 \pm 27.1$ & 0.02 \\
\hline Saturated fat (g) & $24.7 \pm 8.3$ & $22.6 \pm 10.4$ & 0.41 & $27.8 \pm 10.6$ & $20.6 \pm 10.3$ & 0.01 \\
\hline Cholesterol (mg) & $268.8 \pm 162.19$ & $193.3 \pm 111.6$ & 0.05 & $305.7 \pm 215.2$ & $202.0 \pm 104.3$ & 0.03 \\
\hline
\end{tabular}

Values are expressed in mean \pm SD and analyzed. a: Control versus Intervention at baseline; b: Control versus Intervention at 2 months after enrollment.

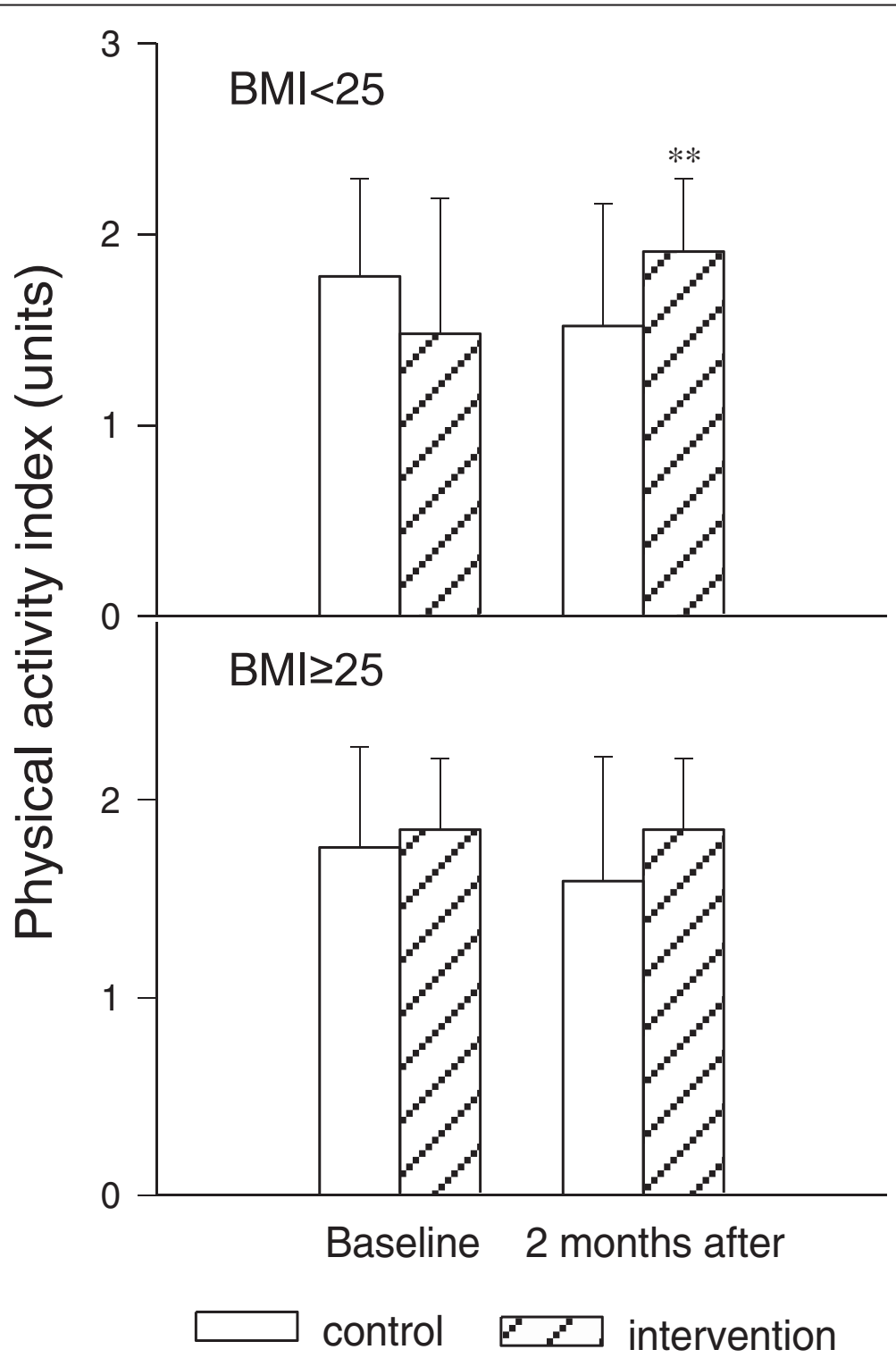

Figure 2 Effect of lifestyle intervention on physical activity index levels in pregnant women with normal or above normal pre-pregnancy BMI at base line and 2 months after enrolment. Upper: normal pre-pregnancy BMI (control $n=27$, intervention $n=30$ ); bottom: above normal pre-pregnancy BMI (control $n=29$, intervention $n=27$ ). Values were expressed in mean $\pm S D$. ${ }^{* *}: p<0.01$ versus control group with corresponding pre-pregnancy BMI. 
and is an unique approach in the literature. Results from this kind of data collection could capture the whole picture of food choice decision-makings in the participants. This is a good indicator that the FCM promoted dietary changes with good understanding of the reasons of participant's food decision making. Women with high pregnancy BMI could have long-term lifestyle habits that influence their food choice, although could be identify through FCM, it might be harder to correct in a limited of time to show significant improvements in gestational weight gain.

Exercise intervention in the literature was either a general encouragement of mild exercise such as walking, or gave verbal or written information on exercise [11-18]. The exercise intervention in this study was a combination of feasible exercise at home and group exercise to strengthen the adherence to the exercise routine. A specifically designed video exercise instruction guided the home exercise which ensured the participant can exercise at the appropriate intensity level on regular basis. This was extremely helpful when the participant could not come to the group exercise due to conflict appointments or weather changes. Weekly group exercise led by a professional trainer helped participants to acquire and validate knowledge and skills for exercise during pregnancy. Group exercise may also help to develop acceptance and adhesion of pregnant women to the healthy lifestyle program. The activity logbook and follow-up visits helped the monitoring of physical activity in participants.

Homogenous recommendation on total calorie intake for normal and overweight pregnant women could be one of reasons for inappropriate GWG and related outcomes in pregnant women with above normal pre-pregnancy BMI. The IOM Food and Nutrition Board published Dietary Reference Intake information for pregnant women in 2006 [23]. Health Canada adapted those recommendations and provided information on key nutrients that are important for maternal and fetal health. The energy requirement recommended for pregnant women with normal pre-pregnancy BMI was $1900 \mathrm{kcal} /$ day in the first trimester, an extra $452 \mathrm{kcal}$ in the second or third trimester [25]. The extra calorie intake was intended to support fetal growth and development. Our study showed that women in the intervention group with normal prepregnancy BMI had a total intake of $2016 \mathrm{kcal} /$ day in the third trimester, which was close to that recommended by the Health Canada for this group of women. As a result, 90\% participants with normal pre-pregnancy BMI obtained weight gain within the recommended limit in the pre-pregnancy BMI category. Women in the control group with normal pre-pregnancy BMI had significantly higher intakes (2 $551 \mathrm{kcal} /$ day in average). The majority of the intake in these women also met the total calorie requirement of Health Canada guidelines for pregnant women. This unnecessary level of total intake could partially contribute to increased GWG, EGWG and offspring birth weight in pregnant women with normal pre-pregnancy BMI without intervention.

Calorie recommendation for overweight or obese pregnant women to achieve the IOM recommended pregnancy weight gain has not been defined. Studies in the past had experienced the same difficulties using lifestyle intervention to achieve proper weight gain in pregnant women with high pre-pregnancy BMI women compared to those with normal pre-pregnancy BMI [14]. Two studies $[13,15]$ specifically targeted pregnant women with higher pre-pregnancy BMI showed successes on weight gain control by setting up meal plans or calorie intake goals. One of the studies reported averages of calorie intake in 1743 and $1784 \mathrm{kcal} /$ day for the second and third trimester in pregnant women in the intervention group with no instructed exercise [13]. The other reported an average of $1900 \mathrm{kcal} /$ day intake with 3-4 times/week walking in pregnant women in the intervention group through pregnancy [15]. These findings suggest that restricted calorie intake could help pregnant women with high -pre-pregnant BMI to achieve recommended GWG. The present study demonstrated that the participants in the intervention group with normal and above normal pre-pregnancy BMI had similar calorie intakes a months after the intervention (2 $016 \mathrm{kcal} / \mathrm{d}$ versus $1986 \mathrm{kcal} / \mathrm{d}$ ). Normal pre-pregnancy BMI, but not above normal BMI, pregnant women receiving the intervention had lower carbohydrate and higher physical activity compared to the control group. The lifestyle intervention reduced EGWG, GWG and birth weight of offspring only in the normal BMI group, but not in high BMI subgroup. It may be speculated that, more intensive intervention to reduce carbohydrate intake and to increase physical activity might be required in order to achieve the goal of normal pregnancy weight gain recommended by the 2009 IOM guidelines [7].

\section{Study limitations}

The sample size of the present study limited the possibility to further divide the study subjects to more detailed subgroups in pre-pregnancy BMI, such as overweight, obese and massive obese, which may weaken the discrimination of the responses from subjects with various intensity of obesity on the lifestyle intervention.

The IOM 2009 guidelines on weight gain recommendations in pregnancy were based on assumptions that a $0.5-2 \mathrm{~kg}$ weight gain in the first trimester [7]. Since some participants had no record on pre-pregnancy weight, their earliest weight in pregnancy $(<10$ weeks of pregnancy) was used as pre-pregnancy weight. This could mildly affect the accuracy of the calculation of total GWG and EGWG. 
Although self-reported food records have been commonly used to collect food intake data, there is a possibility that the women with above normal BMI might underreport their intake. It has been reported in the literature that overweight women tended to underreport their daily intake [30]. This could affect the accuracy of the nutrition intake.

\section{Conclusion and future implementation}

The lifestyle intervention program in this RCT effectively reduced EGWG, GWG and offspring birth weight in pregnant women with normal pre-pregnancy BMI, but not in women with above normal pre-pregnancy BMI. Better adaptation to education on food intake and physical activity may contribute to the weight gain control in normal pre-pregnancy BMI women than in those with above normal pre-pregnancy BMI. Future studies may rationalize the level of carbohydrate intake and physical activity for pregnant women with above normal pre-pregnancy BMI, and further explore the effect of enhanced dietary education and physical activity program on GWG in pregnant women with above normal pre-pregnancy BMI. A qualitative study that explores the barriers of women with above normal pre-pregnancy BMI in achieving recommended gestational weight gain may be necessary to understand this population and developing better clientcentered education tools.

\section{Abbreviations \\ BMI: Body mass index; GWG: Gestational weight gain; EGWG: Excessive gestational weight gain; IOM: Institute of Medicine; RCT: Randomized controlled trial; FCM: Food choice map.}

\section{Competing interests}

The authors declare that there are no competing interests.

\section{Authors' contributions}

Study conception and design, AH, SL,PG, GS, HD, ES, JM, MM, GS. Coordination and implementation of the study AH, GS. Data collection, AH. Data analysis, DJ. Preparation of the manuscript, $\mathrm{AH}$. Editorial assistance, all authors. All authors read and approved the final manuscript.

\section{Acknowledgements}

The authors would like to thank the staff of the Healthy Start for Mom \& Me Prenatal Program in Winnipeg for their help with recruitment, and grant support from the Canadian Institutes of Health Research, the Lawson Foundation and the Public Health Agency of Canada.

\section{Author details}

${ }^{1}$ Departments of Internal Medicine, University of Manitoba, Room 801D, 715 McDermot Ave., Winnipeg, MB R3E 3P4, Canada. ${ }^{2}$ Kinesiology, University of Manitoba, Winnipeg, MB, Canada. ${ }^{3}$ Human Nutritional Sciences, University of Manitoba, Winnipeg, MB, Canada. ${ }^{4}$ Department of Pediatrics and Child Health, University of Manitoba, Winnipeg, MB, Canada. ${ }^{5}$ Obstetrics and Gynecology, University of Manitoba, Winnipeg, MB, Canada.

Received: 5 March 2014 Accepted: 16 September 2014 Published: 24 September 2014

\section{References}

1. Canada H: Canadian Guidelines for Body Weight Classification in Adults (Catalogue H49-179); 2003.
2. Canadian Institute for Health Information: Improving the Health of Canadians; 2004

3. Gilmore J: Body mass index and health. Health Rep Summer 1999, 11:31-43 (Eng). 33-47 (Fre).

4. Lau DC, Douketis JD, Morrison KM, Hramiak IM, Sharma AM, Ur E: Canadian clinical practice guidelines on the management and prevention of obesity in adults and children [summary]. CMAJ 2006, 2007(176):S1-S13.

5. Tjepkema M, Shields M: Canadian community health survey - Measured obesity: adult obesity in Canada. Ottawa, ON: Statistics CanadA; 2005. 82-620MWE2005001.

6. Public Health Agency of Canada: The Canadian Maternity Experiences Survey; 2006.

7. Rasmussen KM, Yaktine AL: Weight Gain during Pregnancy: Reexamining the Guidelines. Washington, D.C: Institute of Medicine and National Research Council of the National Academies. The National Academies Press; 2009.

8. ACOG: Committee opinion: weight gain during pregnancy. Number 548, Jan. 2013. http://www.acog.org/Resources\%20And\%20Publications/Committee\% 200pinions/Committee\%20on\%200bstetric\%20Practice/Weight\%20Gain\% 20During\%20Pregnancy.aspx.

9. Health Canada: Prenatal Nutrition Guidelines for Health Professionals: Gestational Weight Gain; http://hc-sc.gc.ca/fn-an/nutrition/prenatal/ewbambsa-eng.php\#a2.

10. Borberg C, Gillmer MD, Brunner EJ, Gunn PJ, Oakley NW, Beard RW: Obesity in pregnancy: the effect of dietary advice. Diabetes Care 1980, 3:476-481.

11. Olson CM, Strawderman MS, Reed RG: Efficacy of an intervention to prevent excessive gestational weight gain. Am J Obstet Gynecol 2004, 191:530-536.

12. Claesson IM, Sydsjo G, Brynhildsen J, Cedergren M, Jeppsson A, Nyström F, Sydsjö A, Josefsson A: Weight gain restriction for obese pregnant women: a case-control intervention study. BJOG 2008, 115:44-50.

13. Wolff S, Legarth J, Vangsgaard K, Toubro S, Astrup A: A randomized trial of the effects of dietary counseling on gestational weight gain and glucose metabolism in obese pregnant women. Int J Obes (Lond) 2008, 32:495-501.

14. Asbee SM, Jenkins TR, Butler JR, White J, Elliot M, Rutledge A: Preventing excessive weight gain during pregnancy through dietary and lifestyle counseling: a randomized controlled trial. Obstet Gynecol 2009, 113(2 Pt 1):305-312.

15. Mottola MF, Giroux I, Gratton R, Hammond JA, Hanley A, Harris S, McManus R, Davenport MH, Sopper MM: Nutrition and exercise prevent excess weight gain in overweight pregnant women. Med Sci Sports Exerc 2010, 42:265-272.

16. Polley BA, Wing RR, Sims CJ: Randomized controlled trial to prevent excessive weight gain in pregnant women. Int J Obes Relat Metab Disord 2002, 26:1494-1502

17. Gray-Donald K, Robinson E, Collier A, David K, Renaud L, Rodrigues S: Intervening to reduce weight gain in pregnancy and gestational diabetes mellitus in Cree communities: an evaluation. CMAJ 2000, 163:1247-1251.

18. Guelinckx I, Devlieger R, Mullie P, Vansant G: Effect of lifestyle intervention on dietary habits, physical activity, and gestational weight gain in obese pregnant women: a randomized controlled trial. Am J Clin Nutr 2010, 91:373-380.

19. Hui A, Back L, Ludwig S, Gardiner P, Sevenhuysen G, Dean H, Sellers E, McGavock J, Morris M, Bruce S, Murray R, Shen GX: Lifestyle intervention on diet and exercise reduced excessive gestational weight gain in pregnant women under a randomised controlled trial. BJOG 2012, 119:70-77.

20. Hui A, Ludwig S, Gardiner P, Sevenhuysen G, Murray R, Morris M, Shen GX: Report of a pilot study on community - based exercise and dietary intervention during pregnancy. Can J Diabetes 2006, 31:169-175.

21. Sevenhuysen GP, Gross U: Documenting the reasons people have for choosing their food. Asia Pac J Clin Nutr 2003, 12:30-37.

22. Shuaibi AM, Sevenhuysen GP, House JD: Validation of a food choice map with a 3-day food record and serum values to assess folate and vitamin B-12 intake in college-aged women. J Am Diet Assoc 2008, 108:2041-2050.

23. Institute of Medicine: Dietary Reference Intakes: The Essential Guide to Nutrient Requirements; 2006.

24. Canada H: Prenatal Nutrition Guidelines for Health Professionals: gestational weight gain; 2010.

25. Canada H: Prenatal Nutrition Guidelines for Health Professionals - Background on Canada's Food Guide; 2009.

26. Canadian Diabetes Association, Clinical Practice Guidelines Committee, Canadian Diabetes Association: 2008 Clinical Practice Guidelines for the 
Prevention and Management of Diabetes in Canada. Can J Diabetes Care 2008, 32:51:171

27. Williams RL, Creasy RK, Cunningham GC, Hawes WE, Norris FD, Tashiro M: Fetal growth and perinatal viability in California. Obstet Gynecol 1982, 59:624-632.

28. Mottola MF, Davenport MH, Brun CR, Inglis SD, Charlesworth S, Sopper MM: Vo2 peak prediction and exercise prescription for pregnant women. Med Sci Sports Exerc 2006, 38:1389-1395.

29. Gibson RS: Priniciples of Nutritional Assessment. New York: Oxford University Press; 1990.

30. Garriguet D: Under-reporting of energy intake in the Canadian Community Health Survey. Health Rep 2008, 19:37-45.

doi:10.1186/1471-2393-14-331

Cite this article as: Hui et al:: Effects of lifestyle intervention on dietary intake, physical activity level, and gestational weight gain in pregnant women with different pre-pregnancy Body Mass Index in a randomized control trial. BMC Pregnancy and Childbirth 2014 14:331.

\section{Submit your next manuscript to BioMed Central and take full advantage of:}

- Convenient online submission

- Thorough peer review

- No space constraints or color figure charges

- Immediate publication on acceptance

- Inclusion in PubMed, CAS, Scopus and Google Scholar

- Research which is freely available for redistribution 